\title{
Phytoprotection
}

\section{Évaluation au Québec d'un modèle de prédiction de la fin de la période annuelle d'éjection des ascospores du Venturia inaequalis.}

\section{St-Arnaud et P. Neumann}

Volume 71, numéro 1, 1990

URI : https://id.erudit.org/iderudit/705978ar

DOI : https://doi.org/10.7202/705978ar

Aller au sommaire du numéro

Éditeur(s)

Société de protection des plantes du Québec (SPPQ)l

ISSN

0031-9511 (imprimé)

1710-1603 (numérique)

Découvrir la revue

Citer cet article

St-Arnaud, M. \& Neumann, P. (1990). Évaluation au Québec d'un modèle de prédiction de la fin de la période annuelle d'éjection des ascospores du Venturia inaequalis.. Phytoprotection, 71(1), 17-23.

https://doi.org/10.7202/705978ar
Résumé de l'article

Un modèle de prédiction de la fin de la période des éjections d'ascospores du Venturia inaequalis, développé à partir de données recueillies au New Hampshire, a été évalué dans sept localités de pomoculture québécoise, entre 1981 et 1987. Pour Frelighsburg 1983 et Saint-Joseph-du-Lac 1982, à la date à laquelle le modèle a estimé le niveau de $100 \%$ d'ascospores matures, les valeurs observées n'ont pas différé significativement des estimés; dans tous les autres cas, les valeurs observées ont été significativement inférieures aux estimés avec une différence variant entre $4,2 \%$ et $35,4 \%$. Le modèle a aussi prédit trop tôt la fin de la période d'éjection des ascospores dans 12 cas sur 16, alors qu'il restait entre $14 \%$ et $70 \%$ des ascospores dans les pseudothèces. 


\title{
Évaluation au Québec d'un modèle de prédiction de la fin de la période annuelle d'éjection des ascopores du Venturia inaequalis
}

\author{
Marc St-Arnaud \\ Jardin botanique de Montréal, 4101, rue Sherboooke est, \\ Montréal (Québec), Canada H1X 2B2
}

\author{
Peterjurgen Neumann \\ Département des sciences biologiques, Université de Montréal, \\ C.P. 6128, Succursale A, Montréal (Québec), Canada H3C 3J7
}

(Reçu 1989-07-21; accepté 1989-12-12)

Un modèle de prédiction de la fin de la période des éjections d'ascospores du Venturia inaequalis, développé à partir de données recueillies au New Hampshire, a été évalué dans sept localités de pomoculture québécoise, entre 1981 et 1987. Pour Frelighsburg 1983 et Saint-Joseph-du-Lac 1982, à la date à laquelle le modèle a estimé le niveau de $100 \%$ d'ascospores matures, les valeurs observées n'ont pas différé significativement des estimés; dans tous les autres cas, les valeurs observées ont été significativement inférieures aux estimés avec une différence variant entre $4,2 \%$ et $35,4 \%$. Le modèle a aussi prédit trop tôt la fin de la période d'éjection des ascospores dans 12 cas sur 16, alors qu'il restait entre $14 \%$ et $70 \%$ des ascospores dans les pseudothèces.

St-Arnaud, M. et P. Neumann. 1990. Évaluation au Québec d'un modèle de prédiction de la fin de la période annuelle d'éjection des ascospores du Venturia inaequalis. PHYTOPROTECTION 71: 17-23.

A model for forecasting the end of the Venturia inaequalis ascospore ejection period, developed from New Hampshire data, was evaluated in seven apple growing localities in the province of Québec from 1981 to 1987. For Frelighsburg 1983 and Saint-Joseph-du-Lac 1982, at the date estimated by the model for the $100 \%$ mature ascospore level, the observed values were not significantly different from the estimates; in all other cases, the observed values were significantly lower than the estimates with a difference between $4.2 \%$ and $35.4 \%$. The model has also underestimated the duration of the ascospore ejection period in 12 of 16 cases with $14 \%$ to $70 \%$ of the spores still remaining in the asci.

\section{Introduction}

Chaque année, les pomiculteurs dépensent des sommes d'argent considérables pour enrayer le développement de la tavelure du pommier (Venturia inaequalis [Cke.] Wint.). Ce champignon cause des lésions au feuillage et aux autres tissus sensibles des pommiers (Malus pumila Mill.) par le biais d'ascospores et par la suite, de conidies. Les ascospores sont éjectées au printemps durant les périodes de pluie; les éjections s'étendent approximativement entre le débourrement des pommiers et la mi-juin ou la mi-juillet, selon les années. Les conditions qui influencent la maturation et l'éjection des ascospores ont été récemment l'objet de plusieurs études (Gadoury et MacHardy 1982a; Gadoury et al. 1984; James et Sutton 1982a; Jeger 1984; Jeger et
Butt 1983; O'Leary et Sutton 1986; StArnaud et al. 1985).

Ces études ont donné lieu à la présentation de deux modèles d'estimation de la maturité des ascospores. Le premier, construit au New-Hampshire, permet l'estimation du probit de la proportion d'ascospores matures à partir des degrés-jours (base $0^{\circ} \mathrm{C}$ ) totalisés depuis l'apparition des premières ascospores matures dans les asques (Gadoury et MacHardy 1982b). Le second modèle, construit en Caroline du Nord, permet l'estimation du stade de développement des pseudothèces à partir d'une équation basée sur la température moyenne quotidienne ainsi que sur des seuils minimaux d'humidité de l'air et de précipitation; les estimations quotidiennes sont totalisées depuis le $1^{\text {er }}$ février, additionnées à une constante (5) et le logit de la proportion d'ascospores matures est ensuite obtenu à l'aide d'une seconde équation d'estimation (James et Sutton 1982b). Ces deux modèles 
se sont ajoutés à celui déjà construit dans l'état de New York et qui permet l'estimation du probit de la proportion d'ascospores matures à l'aide des degrés-jours (base $0^{\circ} \mathrm{C}$ ) et de la précipitation totalisés depuis la date correspondant à $50 \%$ de défeuillaison des pommiers (Massie et Szkolnik 1974).

Deux de ces équations (James et Sutton 1982b; Massie et Szkolnik 1974) ont déjà été évaluées à l'extérieur de leur aire géographique d'origine et ont alors fourni des estimations biaisées (MacHardy et Gadoury 1985; Sutton et al. 1981). Le troisième modèle (Gadoury et MacHardy 1982b) a donné des estimations valables dans les états de New York et de Pennsylvanie et a servi de base pour l'élaboration d'un modèle de prédiction de la fin de période d'éjection des ascospores (MacHardy et Gadoury 1985). $\mathrm{Ce}$ modèle de prédiction repose sur deux équations: la première permet d'estimer le probit de la proportion d'ascospores matures $(\hat{Y})$ à partir des degrés-jours (base $0^{\circ} \mathrm{C}$ ) totalisés depuis le débourrement des pommiers cv. McIntosh (X):

$$
\hat{\mathrm{Y}}=2,51+0,01 \mathrm{X}
$$

intervalle de confiance de $90 \%: \hat{Y} \pm 0,814$

La seconde équation génère des prédictions d'accumulation de degrés-jours $(\hat{Y})$ à partir du nombre de jours écoulés depuis le 15 mars $(X)$ et est valable pour le sud de l'état du New Hampshire:

$\hat{Y}=1,30+1,17 X+0,0949 X^{2}$

Les auteurs considèrent que la fin de la saison des infections primaires est atteinte lorsque $90 \%$ des ascospores de l'année ont été éjectées. Ce seuil est atteint lorsqu'il est tombé plus de $2,5 \mathrm{~mm}$ de pluie durant les heures de clarté de deux journées différentes, après que le niveau de $100 \%$ d'asques matures ait été estimé par la totalisation des degrés-jours.

Le but de cette étude a donc été d'évaluer les estimations générées par le modèle du New Hampshire dans les conditions climatiques qui prévalent au Québec.

\section{Matériel et méthodes}

Origine et caractéristiques du matériel étudié. Des lots de feuilles de pommier cv.
McIntosh infectées par le $V$. inaequalis ont été récoltées après la défeuillaison automnale dans les localités et pour les années suivantes: Farnham 1981, 1986; Hemmingford 1981, 1986; Île d'Orléans 1981, 1984, 1986; Rougemont 1981, 1984, 1986; SaintJoseph-du-Lac 1981, 1984; Saint-Paul d'Abbotsford 1981. Ces localités sont réparties sur un axe NE-SO de $320 \mathrm{~km}$ et couvrent l'ensemble de l'aire de pomoculture québécoise. Les feuilles ont été entreposées durant chaque hiver en une couche de $5 \mathrm{~cm}$ d'épaisseur, dans chaque localité, sur le sol d'un verger choisi par le Ministère de l'agriculture, des pêcheries et de l'alimentation du Québec pour ses relevés climatologiques; le lot a été placé en un endroit semi-ombragé, exposé aux intempéries et distant d'au plus $3 \mathrm{~m}$ de l'abri pour les instruments météorologiques. Les feuilles ont été retenues à l'aide de cages grillagées $(100 \mathrm{~cm} \times 50 \mathrm{~cm} \times 5 \mathrm{~cm})$.

Détermination de l'état des asques et ascospores. Au printemps suivant la récolte, un échantillon de 20 feuilles a été prélevé chaque semaine à compter de la fonte de la neige couvrant la cage grillagée et jusqu'à la mi-juillet ou jusqu'à l'épuisement des feuilles disponibles. Les feuilles ont été insérées dans un sachet hermétique et placées dans un congélateur $\left(-15^{\circ} \mathrm{C}\right)$ jusqu'à l'examen au microscope. L'état de maturité des asques a été évalué par l'examen hebdomadaire du contenu de 20 pseudothèces par localité (Gadoury et MacHardy 1982 c; St-Arnaud et al. 1985). Des données similaires récoltées précédemment pour les printemps 1981, 1982 et 1983 à Frelighsburg (St-Arnaud et al. 1985) ont aussi été utilisées.

Repère phénologique et données climatiques. Les dates correspondant au stade phénologique du débourrement des pommiers cv. McIntosh, pour chacune des localités et années, ont été obtenues de la Station de recherche en phytotechnie du Ministère de l'agriculture, des pêcheries et de l'alimentation du Québec à Farnham. Les degrés-jours en base $0^{\circ} \mathrm{C}$ (moyenne des températures maximales et minimales quotidiennes de l'air, excluant les moyennes inférieures à $0^{\circ} \mathrm{C}$ ) et la précipitation $(\mathrm{mm})$ ont été obtenues de la même source. Ces données ont été enregistrées constamment sur 
les emplacements de maturation des pseudothèces. Les valeurs ont été obtenues à l'aide d'un thermographe $\left( \pm 0,25^{\circ} \mathrm{C}\right)$ et d'un pluviomètre $( \pm 0,1 \mathrm{~mm})$. Le thermographe était placé à $1 \mathrm{~m}$ du sol, dans un abri pour les instruments météorologiques.

Estimations et analyses statistiques. Les probits des estimés de maturité des ascospores ont été obtenus à l'aide de l'équation 1 et l'intervalle de confiance $90 \%$ des estimés correspond à $\hat{Y} \pm 0,814$ (Gadoury et MacHardy 1982b). Les probits ont été convertis en proportions d'ascospores matures à l'aide d'une table (Fisher et Yates 1957). Comme l'écart-type des estimés était connu, les valeurs observées ont été converties en probits et comparées aux estimés à l'aide d'un test de signification pour une valeur individuelle (Sokal et Rohlf 1981).

Les dates estimées de fin de la période d'éjection des ascospores (90\% d'ascospores éjectées) correspondent à la seconde journée d'au moins $2,5 \mathrm{~mm}$ de pluie durant les heures de clarté, après que le niveau de $100 \%$ d'ascospores matures ait été estimé. Les dates minimales et maximales de l'intervalle de confiance de $90 \%$ pour les dates de fin de la période d'éjection des ascospores sont estimées selon la considération précédente, respectivement après que les bornes supérieures et inférieures de l'intervalle de confiance de $90 \%$ pour la proportion d'ascospores matures aient atteint le niveau de $100 \%$. Les valeurs estimées $(90 \%)$ et observées d'ascospores éjectées ont été comparées à l'aide d'un test pour la différence entre deux proportions (Baillargeon et Rainville 1977).

\section{Résultats et discussion}

L'utilité du modèle de MacHardy et Gadoury (1985) repose essentiellement sur l'équation qui permet d'estimer la proportion d'ascospores matures. Elle dépend aussi de la présomption liant la fin de la période annuelle d'éjection à un niveau de maturation des ascospores, suivi d'un seuil minimal de précipitation. Selon les auteurs du modèle, l'équation servant à prédire les degrés-jours doit être adaptée à chaque nouvelle région à l'aide des données climatiques appropriées. Nous avons donc évalué les estimés de maturité des ascospores et les dates de fin de la période annuelle d'éjection qui en découlaient, à l'aide des degrés-jours réels totalisés pour chaque localité et année. Ces estimés ont été comparés aux proportions d'ascospores matures ainsi qu'aux proportions d'ascospores éjectées observées par l'examen du contenu des pseudothèces.

Selon les localités et les années, le modèle a fourni des estimations variables pour la maturité des ascospores (tableau 1). Pour Saint-Joseph-du-Lac 1982 ainsi que Frelighsburg 1983, à la date correspondant au seuil de $100 \%$ d'ascospores matures, estimée par le modèle, aucune différence significative n'a été obtenue entre la proportion d'ascospores matures observées et la proportion estimée. Cependant, pour toutes les autres régions et années, à la fin de la période d'éjection, le modèle a systématiquement surestimé la maturité des ascospores. Dans tous les cas, à la date correspondant au seuil de $100 \%$ d'ascospores matures estimé par le modèle, les proportions d'ascospores matures observées étaient significativement $(P<0,001)$ inférieures. La différence entre le niveau estimé (100\%) et celui observé a varié entre 4,2\% pour Rougemont 1982 et $35,4 \%$ pour SaintJoseph-du-Lac 1985.

Les pourcentages de l'inoculum annuel d'ascospores matures et leurs intervalles de confiance de $90 \%$, tel qu'estimés par le modèle, ainsi que les observations obtenues par l'examen du contenu des pseudothèces pour Frelighsburg 1983 et l'Île d'Orléans 1985 sont illustrés à la figure 1. Pour Frelighsburg 1983, le taux de maturation des ascospores a été plus élevé que ce qui a été estimé par le modèle entre le début de la période de maturation et le niveau de $90 \%$ d'ascospores matures; ensuite, le taux de maturation a diminué brusquement et en fin de période de maturation, la proportion d'ascospores matures observée n'a pas différé significativement de la proportion estimée par le modèle. Pour l'île d'Orléans 1985 , on remarque une bonne concordance entre les observations et les estimés entre le début de la période de maturation et le niveau de $75 \%$ d'ascospores matures; le taux de maturation diminue ensuite et en fin de période de maturation, les proportions d'ascospores matures observées sont significativement plus faibles que les estimés du 
Tableau 1. Maturation des ascospores du Venturia inaequalis; comparaison entre le niveau de $100 \%$ d'ascospores matures estimé par le modèle de prédiction et la valeur observée pour différentes années et localités de pomoculture québécoise

\begin{tabular}{|c|c|c|c|c|c|c|c|}
\hline \multirow[b]{2}{*}{ Localité } & \multirow[b]{2}{*}{ Date } & \multirow{2}{*}{$\begin{array}{c}\text { Estimé des } \\
\text { ascospores } \\
\text { matures }^{\S} \\
(\%)\end{array}$} & \multicolumn{2}{|c|}{$\begin{array}{c}\text { Intervalle de } \\
\text { confiance de } 90 \%^{+}\end{array}$} & \multirow{2}{*}{$\begin{array}{c}\text { Valeur } \\
\text { observée des } \\
\text { ascospores } \\
\text { matures }^{\ddagger} \\
(\%)\end{array}$} & \multirow[b]{2}{*}{$t^{\rrbracket}$} & \multirow[b]{2}{*}{$P$} \\
\hline & & & minimum & maximum & & & \\
\hline \multirow[t]{3}{*}{ Frelighsburg } & $81-06-03$ & 100 & 99,3 & 100 & 91,4 & $-4,584$ & $<0,001$ \\
\hline & $82-06-07$ & 100 & 99,4 & 100 & 84,0 & $-5,533$ & $<0,001$ \\
\hline & $83-06-13$ & 100 & 99,2 & 100 & 98,1 & $-1,706$ & $>0,05$ \\
\hline \multirow[t]{2}{*}{ Farnham } & $82-06-08$ & 100 & 99,8 & 100 & 79,7 & $-5,657$ & $<0,001$ \\
\hline & $87-06-02$ & 100 & 99,9 & 100 & 75,8 & $-6,433$ & $<0,001$ \\
\hline \multirow{2}{*}{$\begin{array}{l}\text { Saint-Joseph- } \\
\text { du-Lac }\end{array}$} & $82-06-08$ & 100 & 99,7 & 100 & 96,6 & $-1,745$ & $>0,05$ \\
\hline & $85-06-05$ & 100 & 99,3 & 100 & 64,6 & $-5,831$ & $<0,001$ \\
\hline \multirow[t]{3}{*}{ Île d'Orléans } & $82-06-21$ & 100 & 99,9 & 100 & 93,9 & $-4,574$ & $<0,001$ \\
\hline & $85-06-25$ & 100 & 99,9 & 100 & 94,2 & $-4,847$ & $<0,001$ \\
\hline & $87-06-15$ & 100 & 99,9 & 100 & 71,9 & $-6,499$ & $<0,001$ \\
\hline \multirow[t]{2}{*}{ Hemmingford } & $82-06-07$ & 100 & 99,5 & 100 & 94,8 & $-3,525$ & $<0,001$ \\
\hline & $87-06-01$ & 100 & 99,9 & 100 & 80,5 & $-6,302$ & $<0,001$ \\
\hline $\begin{array}{l}\text { Saint-Paul } \\
\text { d'Abbotsford }\end{array}$ & $82-05-31$ & 99,9 & 98,8 & 100 & 90,4 & $-3,547$ & $<0,001$ \\
\hline \multirow[t]{3}{*}{ Rougemont } & $82-06-08$ & 100 & 99,9 & 100 & 95,8 & $-4,592$ & $<0,001$ \\
\hline & $85-06-10$ & 100 & 99,9 & 100 & 92,4 & $-4,926$ & $<0,001$ \\
\hline & $87-06-01$ & 100 & 99,5 & 100 & 83,6 & $-4,870$ & $<0,001$ \\
\hline
\end{tabular}

$\S$ Valeur estimée selon l'équation suivante: $\hat{\mathrm{Y}}=2,51+0,01 \mathrm{X}$ ou $\hat{\mathrm{Y}}$ est le probit de la proportion d'ascospores matures et $\mathrm{X}$, les degrés-jours totalisés depuis le débourrement des pommiers cv. McIntosh (Gadoury et MacHardy 1982b; MacHardy et Gadoury 1985).

† Intervalle de confiance de $90 \%$ pour la valeur estimée de maturité des ascospores, correspondant à $\hat{Y} \pm 0,814$ (Gadoury et MacHardy 1982b).

¥Valeurs obtenues par l'examen périodique du contenu d'un échantillon de pseudothèces.

I $t$ de Student pour la différence entre la valeur observée et l'estimé de maturation des ascospores ainsi que son niveau de signification (Sokal et Rohlf 1981); les pourcentages ont été préalablement normalisés par la transformation probit. $\mathrm{H}_{0}$ : valeur observée $=$ valeur estimée; $\mathrm{H}_{1}$ : valeur observée $\neq$ valeur estimée.

modèle. Pour les autres régions et années, les valeurs observées d'ascospores matures se sont comportées, face aux estimés générés par le modèle, de façon similaire ou intermédiaire aux résultats présentés à la figure 1 .

Le fait que le modèle du New Hampshire génère souvent des estimations biaisées au Québec indique que celui-ci ne tient vraisemblablement pas compte de toutes les variables qui peuvent influencer la maturation des ascospores du Venturia inaequalis. Ce modèle est basé sur la présomption que le seul facteur limitant la maturation des ascospores en milieu naturel serait la température. Wilson (1928) et plus récemment
James et Sutton (1982a) ont rapporté que l'humidité des feuilles dans lesquelles se forment les pseudothèces a une influence déterminante sur le développement des ascocarpes. De plus, James et Sutton (1982a) ont constaté qu'en conditions naturelles comme en laboratoire, l'humectation des feuilles formant la litière du verger constitue le principal facteur limitant la maturation des ascospores. Il serait donc possible qu'au Québec, comme en Caroline du nord (James et Sutton 1982a), d'autres variables que la température doivent être aussi considérées pour estimer la maturation des ascospores. Cela pourrait expliquer les taux de maturation différents observés au Québec par rapport aux estimés du modèle. 

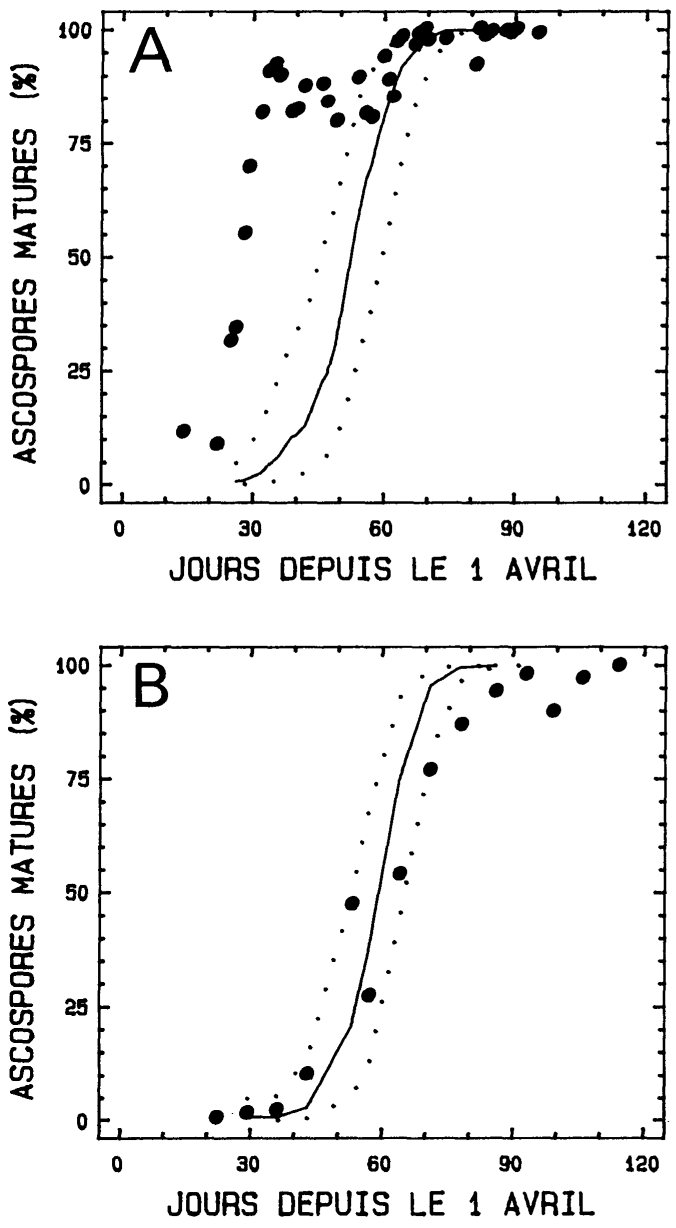

Figure 1. Maturation des ascospores du Venturia inaequalis. Le trait plein (-) représente le pourcentage estimé par le modèle de prédiction alors que les traits pointillés (...) représentent les limites de l'intervalle de confiance au seuil de $90 \%$; les cercles pleins $(\bullet)$ représentent les pourcentages évalués par l'examen du contenu des pseudothèces. A) Frelighsburg 1983; B) Île d'Orléans 1985.

Selon les localités et les années, le modèle a aussi souvent fourni des dates hâtives de fin de saison d'éjection des ascospores (tableau 2). Pour Frelighsburg 1981 et 1983, Saint-Joseph-du-Lac 1982 ainsi que Hemmingford 1982, la proportion d'ascospores éjectées estimée par le modèle $(90 \%)$ n'a pas été significativement plus élevée que la valeur observée, au moins pour la date la plus tardive de l'intervalle de confiance de $90 \%$ pour les dates de fin de la période d'éjection des ascospores. Cependant, à Saint-Joseph-du-Lac 1982, la date la plus hâtive de l'intervalle de confiance correspondait à une valeur observée de $52 \%$ d'ascospores éjectées, soit $48 \%$ des ascospores encore disponibles pour des éjections ultérieures. Pour toutes les régions et années, aux dates minimales et maximales de l'intervalle de confiance, la valeur observée d'ascospores éjectées a été significativement $(P<0,001)$ inférieure à la valeur estimée par le modèle. À Farnham 1982 et à Saint-Paul d'Abbotsford 1982, il restait encore plus de $31 \%$ à $44 \%$ des ascospores non-éjectées; cela situerait la date de fin de la période d'éjection estimée par le modèle dans la portion centrale de la courbe d'éjection (MacHardy et Gadoury 1985), correspondant à la phase accélérée d'éjection des ascospores.

En 1985 et particulièrement en 1987 , le modèle a systématiquement surestimé la proportion d'ascospores éjectées en fin de saison et a prédit la fin de la période d'éjection alors qu'il restait entre $17 \%$ et $19 \%$ (Île d'Orléans 1985) et jusqu'à $70 \%$ (Farnham 1987) des ascospores dans les pseudothèces. L'éjection en elle-même, bien qu'influencée par la pluie et la lumière (Brook 1969; Hirst et Stedman 1961, 1962; Miller et Waggoner 1958; St-Arnaud et al. 1984), n'est possible qu'en présence d'ascospores mûres dans les asques. Les estimations précoces de fin de saison d'éjection, générées par le modèle, sont donc vraisemblablement causées par une mauvaise estimation du taux de maturation des ascospores.

Actuellement, l'utilisation de ce modèle de prédiction ne semble donc pas appropriée pour l'aire géographique étudiée au Québec. Il sera vraisemblablement nécessaire d'inclure d'autres variables ayant une influence sur la maturation ou l'éjection des ascospores, telles que l'humectation des feuilles formant la litière du verger, la pluviométrie et la luminosité, dans une équation similaire afin de pouvoir générer des estimations stables dans les régions où la température ne serait pas le seul facteur limitant la maturation des ascospores.

Les auteurs remercient sincèrement messieurs M. Mailloux, J. Touzain et C. Bouchard du Ministère de l'agriculture, des pêcheries et de l'alimentation du Québec pour les récoltes hebdomadaires d'échantillons de feuilles, mesdames C. Trickey et M. Phaneuf pour leur travail technique, ainsi que madame H. Levert pour la dactylographie du manuscrit. 
Tableau 2. Fin de la période d'éjection des ascospores du Venturia inaequalis; comparaison entre le niveau de $90 \%$ d'ascospores éjectées estimé par le modèle de prédiction et la valeur observée pour différentes années et localités de pomoculture québécoise

\begin{tabular}{|c|c|c|c|c|c|c|c|c|c|c|}
\hline \multirow[b]{2}{*}{ Localité } & \multicolumn{10}{|c|}{$\begin{array}{l}\text { Intervalle de confiance de } 90 \% \text { des dates estimées de fin de la période d'éjection des ascospores } \\
\text { Minimum }\end{array}$} \\
\hline & Date $^{\S}$ & $\begin{array}{c}\text { Valeur } \\
\text { estimée } \\
(\%)\end{array}$ & $\begin{array}{c}\begin{array}{c}\text { Valeur } \\
\text { observée }^{+} \\
(\%)\end{array} \\
\end{array}$ & $Z^{\ddagger}$ & $P$ & Date & $\begin{array}{c}\text { Valeur } \\
\text { estimée } \\
(\%)\end{array}$ & $\begin{array}{c}\text { Valeur } \\
\text { observée } \\
(\%)\end{array}$ & $Z$ & $P$ \\
\hline Frelighsburg & $\begin{array}{l}81-05-30 \\
82-06-20 \\
83-06-27\end{array}$ & $\begin{array}{l}90 \\
90 \\
90\end{array}$ & $\begin{array}{l}84 \\
82 \\
94\end{array}$ & $\begin{array}{r}10,955 \\
14,607 \\
-7,303\end{array}$ & $\begin{array}{l}<0,001 \\
<0,001 \\
>0,05\end{array}$ & $\begin{array}{l}81-06-10 \\
81-06-20 \\
83-06-27\end{array}$ & $\begin{array}{l}90 \\
90 \\
90\end{array}$ & $\begin{array}{l}95 \\
82 \\
94\end{array}$ & $\begin{array}{r}-9,129 \\
14,607 \\
-7,303\end{array}$ & $\begin{array}{l}>0,05 \\
<0,001 \\
>0,05\end{array}$ \\
\hline Farnham & $\begin{array}{l}82-06-16 \\
87-05-29\end{array}$ & $\begin{array}{l}90 \\
90\end{array}$ & $\begin{array}{l}56 \\
30\end{array}$ & $\begin{array}{r}62,078 \\
109,549\end{array}$ & $\begin{array}{l}<0,001 \\
<0,001\end{array}$ & $\begin{array}{l}82-06-19 \\
87-06-14\end{array}$ & $\begin{array}{l}90 \\
90\end{array}$ & $\begin{array}{r}69 \\
-9\end{array}$ & $\begin{array}{c}38,342 \\
-\end{array}$ & $\begin{array}{c}<0,001 \\
-\end{array}$ \\
\hline $\begin{array}{l}\text { Saint-Joseph- } \\
\text { du-Lac }\end{array}$ & $\begin{array}{l}82-06-20 \\
85-06-06\end{array}$ & $\begin{array}{l}90 \\
90\end{array}$ & $\begin{array}{l}52 \\
57\end{array}$ & $\begin{array}{l}69,381 \\
60,252\end{array}$ & $\begin{array}{l}<0,001 \\
<0,001\end{array}$ & $\begin{array}{l}82-06-21 \\
85-06-13\end{array}$ & $\begin{array}{l}90 \\
90\end{array}$ & $\begin{array}{l}95 \\
75\end{array}$ & $\begin{array}{r}-9,129 \\
27,387\end{array}$ & $\begin{array}{l}>0,05 \\
<0,001\end{array}$ \\
\hline Île d'Orléans & $\begin{array}{l}82-06-20 \\
85-06-18 \\
87-06-08\end{array}$ & $\begin{array}{l}90 \\
90 \\
90\end{array}$ & $\begin{array}{l}86 \\
81 \\
29\end{array}$ & $\begin{array}{r}7,303 \\
16,432 \\
111,345\end{array}$ & $\begin{array}{l}<0,001 \\
<0,001 \\
<0,001\end{array}$ & $\begin{array}{l}82-06-23 \\
85-06-24 \\
87-06-19\end{array}$ & $\begin{array}{l}90 \\
90 \\
90\end{array}$ & $\begin{array}{l}- \\
83 \\
56\end{array}$ & $\begin{array}{c}- \\
12,781 \\
62,078\end{array}$ & $\begin{array}{c}- \\
<0,001 \\
<0,001\end{array}$ \\
\hline Hemmingford & $\begin{array}{l}82-06-14 \\
87-05-31\end{array}$ & $\begin{array}{l}90 \\
90\end{array}$ & $\begin{array}{l}92 \\
39\end{array}$ & $\begin{array}{r}-3,652 \\
93,117\end{array}$ & $\begin{array}{l}>0,05 \\
<0,001\end{array}$ & $\begin{array}{l}82-06-14 \\
87-06-03\end{array}$ & $\begin{array}{l}90 \\
90\end{array}$ & $\begin{array}{l}92 \\
56\end{array}$ & $\begin{array}{r}-3,652 \\
62,078\end{array}$ & $\begin{array}{l}>0,05 \\
<0,001\end{array}$ \\
\hline $\begin{array}{l}\text { Saint-Paul } \\
\text { d'Abbotsford }\end{array}$ & $82-06-02$ & 90 & 61 & 52,949 & $<0,001$ & $82-06-02$ & 90 & 61 & 52,949 & $<0,001$ \\
\hline Rougemont & $\begin{array}{l}82-06-19 \\
85-06-06 \\
87-06-08 \\
\end{array}$ & $\begin{array}{l}90 \\
90 \\
90 \\
\end{array}$ & $\begin{array}{l}80 \\
64 \\
41 \\
\end{array}$ & $\begin{array}{l}18,258 \\
47,471 \\
89,465 \\
\end{array}$ & $\begin{array}{l}<0,001 \\
<0,001 \\
<0,001 \\
\end{array}$ & $\begin{array}{l}82-06-19 \\
85-06-13 \\
87-06-09 \\
\end{array}$ & $\begin{array}{l}90 \\
90 \\
90 \\
\end{array}$ & $\begin{array}{l}80 \\
74 \\
41 \\
\end{array}$ & $\begin{array}{l}18,258 \\
29,213 \\
89,465\end{array}$ & $\begin{array}{l}<0,001 \\
<0,001 \\
<0,001\end{array}$ \\
\hline
\end{tabular}

$\S$ Valeurs extrêmes de l'invervalle de confiance pour les dates de fin de la période d'éjection des ascospores (90\% d'ascospores éjectées); les dates minimales et maximales correspondent à la seconde journée d'au moins $2,5 \mathrm{~mm}$ de pluie durant les heures de clarté, après que respectivement les bornes supérieure ou inférieure de l'intervalle de confiance $(90 \%)$ de l'estimé des ascospores matures aient atteint le niveau de $100 \%$.

$\dagger$ Valeurs obtenues par l'examen périodique du contenu d'un échantillon de pseudothèces.

$\ddagger Z$ pour la différence entre deux proportions et son niveau de signification (Baillargeon et Rainville 1977). $\mathrm{H}_{0}:$ proportion estimée $=$ proportion observée; $\mathrm{H}_{1}$ : proportion estimée $>$ proportion observée.

I Résultat non-disponible. 


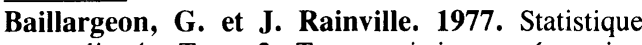
appliquée, Tome 2; Tests statistiques, régression et corrélation. $2^{\mathrm{e}}$ éd. Les Éditions SMG, TroisRivières, 594 pp.

Brook, P.J. 1969. Effects of light, temperature and moisture on release of ascospores by Venturia inaequalis (Cke.) Wint. N. Z. J. Agric. Res. 12: 214-227.

Fisher, R.A. et F. Yates. 1957. Statistical tables for biological, agricultural and medical research. $5^{\mathrm{e}}$ éd. Oliver and Boyd, Edinburgh. $136 \mathrm{pp}$.

Gadoury, D.M. et W.E. MacHardy. 1982a. Effects of temperature on the development of pseudothecia of Venturia inaequalis. Plant Dis. 66: 464-468.

Gadoury, D.M. et W.E. MacHardy. 1982b. A model to estimate the maturity of ascospores of Venturia inaequalis. Phytopathology 72: 901-904.

Gadoury, D.M. et W.E. MacHardy. 1982c. Preparation and interpretation of squash mounts of pseudothecia of Venturia inaequalis. Phytopathology 72: 92-95.

Gadoury, D.M., W.E. MacHardy et C.C. Hu. 1984. Effect of temperature during ascus formation and frequency of ascospore discharge on pseudothecial development of Venturia inaequalis. Plant Dis. 68: 223-225.

Hirst, J.M. et O.J. Stedman. 1961. The epidemiology of apple scab (Venturia inaequalis (Cke.) Wint.). I. Frequency of airborne spores in orchards. Ann. Appl. Biol. 49: 290-305.

Hirst, J.M. et O.J. Stedman. 1962. The epidemiology of apple scab (Venturia inaequalis (Cke.) Wint.). II. Observations on the liberation of ascospores. Ann. Appl. Biol. 50: 525-550.

James, J.R. et T.B. Sutton. 1982a. Environment factors influencing pseudothecial development and ascospore maturation of Venturia inaequalis. Phytopathology 72: 1073-1080.

James, J.R. et T.B. Sutton. 1982b. A model for predicting ascospore maturation of Venturia inaequalis. Phytopathology 72: 1081-1085.

Jeger, M.J. 1984. Overwintering of Venturia inaequalis in relation to lesion intensity on leaf surfaces, and leaf surface exposed. Trans. Br. Mycol. Soc. 83: 495-500.
Jeger, M.J. et D.J. Butt. 1983. Overwintering of Venturia inaequalis the causal agent of apple scab in relation to weather. Ann. Appl. Biol. 103: 201-218.

MacHardy, W.E. et D.M. Gadoury. 1985. Forecasting the seasonal maturation of ascospores of Venturia inaequalis. Phytopathology $75: 381-385$.

Massie, L.B. et M. Szkolnik. 1974. Prediction of ascospore maturation of Venturia inaequalis utilizing cumulative degree days. Proc. Am. Phytopathol. Soc. 1: 140 (résumé).

Miller, P.M. et P.E. Waggoner. 1958. Dissemination of Venturia inaequalis ascospores. Phytopathology 48: 416-419.

O'Leary, A.L. et T.B. Sutton. 1986. The influence of temperature and moisture on the quantitative production of pseudothecia of Venturia inaequalis. Phytopathology 76: 199-204.

St-Arnaud, M., L.J. Coulombe, P. Neumann et A. Jacob. 1984. Étude de la maturation et de l'éjection des ascospores de Venturia inaequalis (Cke.) Wint. en conditions naturelles. Pages 103-111 in C. Vincent et N. Bostonian (réd.). La phytoprotection des vergers de pommiers au Québec. Bull. tech. 19, Sta. Rech. Agric. Can., Saint-Jean-surRichelieu.

St-Arnaud, M., L.J. Coulombe, P. Neumann et A. Jacob. 1985. La maturation et l'éjection des ascospores du Venturia inaequalis à Frelighsburg (Québec) en relation avec la température et la pluie. Phytoprotection 66: 153-161.

Sokal, R.R. et F.J. Rohlf. 1981. Biometry. The principles and practice of statistics in biological research. $2^{\mathrm{e}}$ éd. W.H. Freeman and Co., N.Y. $859 \mathrm{pp}$.

Sutton, T.B., J.R. James et J.F. Nardacci. 1981. Evaluation of a New York ascospore maturity model for Venturia inaequalis in North Carolina. Phytopathology 71: 1030-1032.

Wilson, E.E. 1928. Studies of the ascigerous stage of Venturia inaequalis (Cke.) Wint. in relation to certain factors of the environment. Phytopathology 18: $375-418$. 